\title{
Vitiligo Improvement in Patient with Crohn's Disease Undergoing Treatment with Infliximab
}

\section{Cardoso Mateus Ricardo ${ }^{1 *}$, Tavares Isabela Boulhosa ${ }^{1}$, Cordeiro Karina Ferreira ${ }^{1}$ and Campos Luciana Teixeira ${ }^{2}$}

${ }^{1}$ Medical Student at Centro Universitário de Brasília - UniCEUB, Faculdade de Ciências da Educação e Saúde - FACES, Brasília, Distrito Federal, Brazil

${ }^{2}$ Professor at UniCEUB e Master in Health Sciences, Gastroenterologist at Instituto Hospital de Base do Distrito Federal - IHB-DF, Brasília, Distrito

Federal, Brazil

*Corresponding Author: Cardoso Mateus Ricardo, Medical Student at

Centro Universitário de Brasília - UniCEUB, Faculdade de Ciências da

Educação e Saúde - FACES, Brasília, Distrito Federal, Brazil.
Received: November 10, 2020

Published: November 28, 2020

(C) All rights are reserved by Cardoso Mateus

Ricardo., et al.

\begin{abstract}
Introduction: Vitiligo is a skin disease that causes depigmentation due to the destruction of epidermal melanocytes. The etiology of vitiligo is unknown, and it is mainly related to an autoimmune factor, coursing with imbalance of cytokines in the injured areas. Infliximab is an anti-TNF- $\alpha$ antibody that prevents the binding of TNF- $\alpha$ to its receptors, this can cause both a protective effect and an inducing effect on the autoimmunity of vitiligo.

Objective: Report a case of improvement of vitiligo after treatment with Infliximab and review the existing literature on this subject.

Method: Case report selected due the improvement of vitiligo after the use of Infliximab by means of the analysis of medical records, followed by a bibliographic review in textbooks and Pubmed and Google Scholar databases, which results in a qualitative and exploratory analysis of the literature.

Results: Description of the improvement of a patient with vitiligo after six months of treatment with Infliximab used for Crohn's disease.

Conclusion: Anti-TNF- $\alpha$ agents have been a promising method for the treatment of vitiligo, especially in refractory cases. Infliximab, however, has presented controversial results in the literature, such as aggravation of the condition or onset cases. The results of this report raises suspicions about the effects of TNF- $\alpha$ inhibition of vitiligo's pathophysiology, therefore the role of Infliximab as a treatment form remains questionable.
\end{abstract}

Keywords: Vitiligo; Infliximab; Crohn's Disease; Case Reports; Tumor Necrosis Factor-Alpha

\section{Abbreviations}

TNF- $\alpha$ : Tumor Necrosis Factor-Alpha; HIV: Human Immunodeficiency Virus; IL-6: Interleukin 6; IL-1: Interleukin 1; TGF- $\beta$ : Transforming Growth Factor Beta; TNFR 1: Tumor Necrosis Factor Receptor 1; TNFR 2: Tumor Necrosis Factor Receptor 2; Tregs: Regulatory T Cell; NF-jB: Nuclear Factor Kappa B

\section{Introduction}

Vitiligo is a skin disease that causes depigmentation due to the destruction of melanocytes in the epidermis [1]. It affects $0.5-2 \%$ of the world population, without significant variations between sex and race. Vitiligo is associated with autoimmune diseases such as Graves' disease, Hashimoto's thyroiditis, Diabetes Mellitus, and 
psoriasis [1-3]. The etiology of vitiligo is not fully established: it has a possible genetic factor and is usually related to an abnormal immune response $[1,2,4,5]$.

The evolution of the disease is unpredictable. There are greater chances of spontaneous repigmentation in photo-exposed areas with hair follicles, due the activation and migration of melanocytes from hair follicles to depigmented skin. Although it does not affect individuals' survival, vitiligo can greatly impair their psychology and emotions due to the possible disfigurement and embarrassment caused by lesions [2,3].

Treatment often has unpredictable and frustrating results. There are several therapeutic options according to the severity and evolution of lesions, such as corticosteroids, psoralens, immunomodulators, psorasol, and psoralen-ultraviolet $A[2,3,6]$. In resistant cases can be performed grafts, depigmentation and camouflage. Procedures that cause superficial abrasion of the skin, such as dermabrasion, laser and friction, have been tested as new therapeutic options due to their properties to induce the activation and migration of melanocytes from hair follicles [6].

The anti-TNF- $\alpha$ is a class of monoclonal antibodies developed for the treatment of several autoimmune diseases. However, they are not commonly used for treatment of vitiligo, on account of several reports of hypopigmented lesions as a side effect of the use of this drug class [7]. In this report, we present a case of unusual improvement in vitiligo after a treatment with Infliximab used for Crohn's disease.

\section{Methodology}

This is a case report of a 44-year-old male patient, selected by means of an analysis of his medical record after the vitiligo improvement posterior to the use of Infliximab. The literature was reviewed in textbooks and Pubmed and Google Scholar databases, thereupon a qualitative and exploratory analysis was conducted.

The descriptors used on the databases were: vitiligo Infliximab and Crohn's disease, vitiligo and Infliximab, treatment of vitiligo, pathophysiology of vitiligo, vitiligo and anti-TNF- $\alpha$ agents, vitiligo, Infliximab.

The research was conducted according to Resolution 466/2012, respecting the patient's autonomy and dignity and registering his willingness to contribute and remain in the research through the free and informed consent form (TLCE). The principles of beneficence and non-maleficence were guaranteed, and the study progressed in accordance with current legal precepts and with the guidelines of the National Committee of Ethics in Research (CONEP).

\section{Case Report}

Male patient, 44 years old, diagnosed with Crohn's disease nine years ago, with several stenosing complications, also presents depression and vitiligo (Figure 1 and 2). He has a history of enterectomy with excision of $60 \mathrm{~cm}$ and use of terminal ileostomy, has smoked for five years, ceased four years ago. Presents negative serology for hepatitis B, HIV, hepatitis C, tuberculosis, and syphilis. The medications used include azathioprine $200 \mathrm{mg} /$ day, prednisone $40 \mathrm{mg} /$ day, and sertraline $100 \mathrm{mg} /$ day. The treatment started with Infliximab $5 \mathrm{mg} / \mathrm{kg}$ in January 2017 and after six months, he noticed an improvement in the hypopigmented skin lesions, with pigment islets on the face, forearm and hands (Figure 3 and 4), despite the absence of a specific treatment for vitiligo in the period. Currently, he continues the use of Infliximab, with marked regression of the lesions.

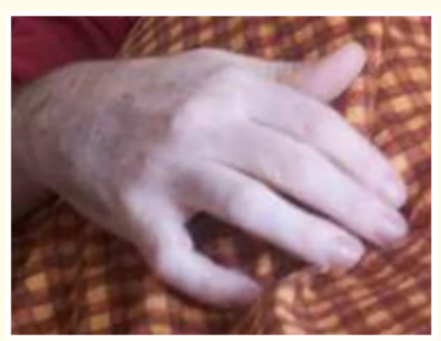

Figure 1: Hand lesions before the use of infliximab

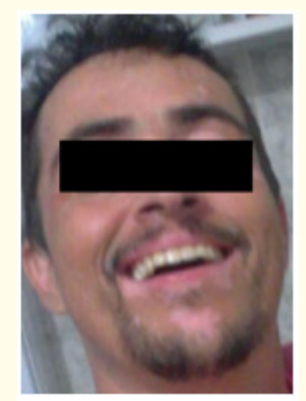

Figure 2: Face lesions before the use of infliximab. 


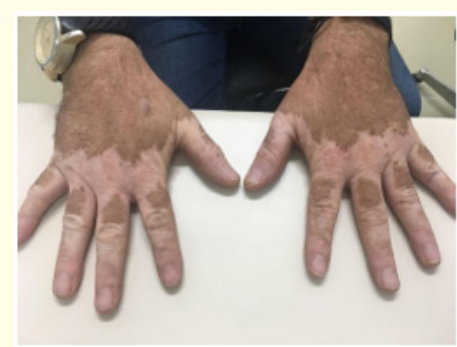

Figure 3: Improvement of hand lesions, 6 months after start of infliximab.

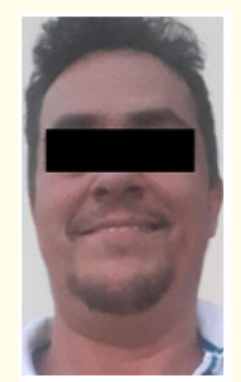

Figure 4: Improvement of face lesions, 6 months after start of infliximab.

\section{Discussion}

In the case reported, hypopigmented lesions were clinically improved six months after initiating the treatment with Infliximab, and progressively improved with the maintenance of medication.

Infliximab is a monoclonal and chimeric anti-TNF- $\alpha$ antibody, $75 \%$ human and $25 \%$ murine, which binds to TNF- $\alpha$ with great affinity with its soluble and transmembrane form, preventing the binding of TNF- $\alpha$ to its receptors. It is commonly used in several diseases, such as ulcerative colitis, rheumatoid arthritis, sarcoidosis, psoriasis, and Crohn's disease [1-3,8]. When Infliximab binds to TNF- $\alpha$, it induces complement-dependent and cell-mediated lysis, capable of generating cell lysis due to cytotoxicity, with the ability to kill specific populations of cells [9].

Recent studies have shown stabilization or regression of active vitiligo with the use of anti-TNF- $\alpha$ substances, mainly with etanercept, in an interval from 2 months to 2 years of treatment, with varying doses [7]. There is a report of improvement in vitiligo with the use of 350 mg of Infliximab: about $30 \%$ of hypopigmented areas improved six months after the beginning of treatment, a response time similar to the case reported here $[3,7]$.

In contrast, several cases of aggravated or onset vitiligo have also been reported in patients using anti-TNF- $\alpha$ agents, especially Infliximab and Adalimumab. Hypochromic or acromic lesions increased after 2 - 8 months of start of the medications. Most cases evolved with partial repigmentation after withdrawal of medication and use of specific treatment for vitiligo $[1,2,8,10]$.

The supposed immunological mechanism that destroys melanocytes is the imbalance between the effect of inhibitory cytokines, such as TNF $\alpha$, IL- 6 , IL-1, TGF- $\beta$, and promoters of melanogenesis, which is reinforced by the findings of overexpression of TNF- $\alpha$ and IL-6 in the injured areas and overexpression of stimulating factors in the perilesional areas and healthy skin $[1,10]$.

TNF- $\alpha$ is an inflammatory cytokine produced mostly by macrophages and activated T cells. Their actions are mediated by the connection with their receptors, TNFR1 and TNFR2. TNFR1 acts primarily in the activation of caspase-dependent apoptosis 3. TNFR2 is rarer, expressed only by some types of cells, such as lymphocytes and melanocytes. It acts activating and stimulating the proliferation of $\mathrm{T}$ cells and selecting regulatory T cells (Tregs) $[1,7]$.

The proinflammatory activity of TNF- $\alpha$ derives predominantly from the activation of cytokines IL- 1 and IL- 6 and from several nuclear transcription factors, such as NF-jB. In addition, it directly influences melanogenesis, due to the paracrine inhibition of the differentiation of melanocytic cells $[3,10]$. Thus, TNF- $\alpha$ plays a fundamental role in the onset of vitiligo because it stimulates the development of cytotoxic T lymphocytes that destroy melanocytes. These lymphocytes (i) maintain the inflammatory process, (ii) expand and perpetuate lesions due to increased expression of interferon-associated with epithelium depigmentation-and (iii) block repigmentation by differentiating stem cells [7].

Nevertheless, since the TNF- $\alpha$ stimulates Tregs, which suppresses the action of autoreactive T cells derived from the cytotoxic action of TCD8 cells in melanocytes, TNF- $\alpha$ acquires a double role of protector or inducer in the pathophysiology of vitiligo, when it exerts an activation and proliferation function on cytotoxic T cell [7]. 


\section{Conclusion}

Anti-TNF- $\alpha$ agents have been investigated as a promising method for the treatment of vitiligo, especially in refractory cases. Infliximab, however, presents controversial results in the literature, with aggravation or onset cases. The results of this report raise suspicions about the effects of TNF- $\alpha$ inhibition on the pathophysiology of vitiligo, and the role of Infliximab as a form of treatment remains questionable. Further studies may elucidate the function of TNF- $\alpha$ in vitiligo and investigate the use of Infliximab and other anti-TNF- $\alpha$ agents as specific or adjuvant therapy for vitiligo.

\section{Conflict of Interest}

There is no conflict of interest.

\section{Search Limits}

Research was conducted only with a participant, preventing the generalization of the results for the general population. In addition, there was no previous follow-up of vitiligo, making it impossible to quantify the improvement in hypopigmentary lesions. The duration of the case report was 3 years, not allowing a description of the evolution of the lesions in the long term. However, the reported case deserves attention due to the evident improvement of vitiligo and can serve as a reference to the future observations.

\section{Bibliography}

1. Luber RP., et al. "New onset vitiligo following commencement of Infliximab in Crohn disease". Journal of Internal Medicine 47.8 (2017): 972-973.

2. Ryu T H., et al. "A Type II Segmental Vitiligo Developed under Infliximab Treatment for Ulcerative Colitis". A Dermatologist 29.6 (2017): 826-827.

3. Simón J A and Burgos-Vargas R. "Vitiligo improvement in a patient with ankylosing spondylitis treated with Infliximab". Dermatology 216.3 (2008): 234-235.

4. Lee S H., et al. "Psoriasis, Vitiligo and Crohn's Disease Co-Existingin a Single Patient: A Variant Type of Multiple Autoimmune Syndrome?" A Dermatologist 29.6 (2017): 782-785.

5. Lv Y., et al. "Use of anti-tumor necrosis factor agents: a possible therapy for vitiligo". Medical Hypotheses 72.5 (2009): 546-547.

6. Ramírez-Hernandez M., et al. "Infliximab-induced vitiligo". Dermatology 210.1 (2005): 79-80.
7. Webb KC., et al. "Tumour necrosis fator- $\alpha$ inhibition can stabilize disease in progressive vitiligo". British Journal of Dermatology 173.3 (2015): 641-650.

8. Posada C., et al. "Vitiligo during treatment of Crohn's disease with adalimumab: adverse effect or co-occurrence". Case Reports in Dermatology 3.1 (2011): 28-31.

9. Bellet J S and Prose NS. "Vitiligo em crianças: uma revisão de classificação, hipóteses sobre patogênese e tratamento". Anais Brasileiros de Dermatologia 80.6 (2005): 631-636.

10. Carvalho CLDB and Ortigosa LCM. "Segmental vitiligo after Infliximab use for rheumatoid arthritis-A case report". Anais Brasileiros de Dermatologia 89.1 (2014): 154-156.

\section{Assets from publication with us}

- Prompt Acknowledgement after receiving the article

- Thorough Double blinded peer review

- Rapid Publication

- Issue of Publication Certificate

- High visibility of your Published work

Website: www.actascientific.com/

Submit Article: www.actascientific.com/submission.php Email us: editor@actascientific.com

Contact us: +919182824667 